\title{
Homogenization of the elastic behavior of a layer-to-layer angle-interlock composite
}

\author{
Patrick Lapeyronnie ${ }^{a}$, Philippe Le Grognec ${ }^{\mathrm{a}, \Uparrow}$, Christophe Binétruy ${ }^{\mathrm{a}}$, François Boussu ${ }^{\mathrm{b}}$ \\ ${ }^{a}$ Ecole des Mines de Douai, Polymers and Composites Technology E Mechanical Engineering Department, 941 rue Charles Bourseul - BP 10838,59508 Douai Cedex, France \\ ${ }^{\mathrm{b}}$ ENSAIT, GEMTEX (Laboratory of Engineering on Textile Materials), 2 Allée Louise et Victor Champier, 59056 Roubaix, France
}

\begin{abstract}
Composite materials reinforced with 3D layer-to-layer angle-interlock fabrics are increasingly employed due to their significant resistance to delamination and impact damage, which is not observed in classical 2D laminated composites. However, the prediction of the mechanical behavior of such composites is challenging due to the intricate fibrous architecture. The structure is intimately linked to its history of manufacturing which induces changes in the reinforcement geometry. The purpose of this work is to assess the equivalent membrane and bending elastic moduli of the shell-type structure by an asymptotic homogenization procedure on a periodic unit cell, in the framework of the Love-Kirchhoff plate theory. A specific Python program using Abaqus software package is developed, allowing for parameterized geometrical modeling and mechanical analysis in a systematic and efficient way. This modeling and simulation tool enables to consider the real composite architecture after infusion and the yarn damage during weaving. The effective properties are finally validated using numerical computations on 3D heterogeneous plates and by comparison with experimental tests.
\end{abstract}

\section{Introduction}

3D reinforcements have been studied for many years in replacement of laminate structures in composite materials. For instance, $3 \mathrm{D}$ interlock geometrical arrangements seem to be a more suitable solution to the delamination and impact damage, among other options, than a classical stacking of 2D plies. Accordingly, 3D woven fabrics may substitute 2D weaves in many applications where they are not appropriate anymore. Indeed, numerous advantages of 3D reinforced composites have already been pointed out. Complex near-net-shape fabrics can be manufactured with better mechanical properties and a decrease of the cutting needs as well as a reduction of the manufacturing and material costs [1]. They also display a better ballistic and low speed impact resistance with improved post-impact properties and a good resistance to crack propagation [2]. Cox and Dadkhah put forward a better delamination resistance, higher ultimate tensile and compressive strains and a good formability, compared to 2D-ply based laminates [3]. Kuo et al. pointed out the almost elimination of weak damaged planes by dissipating loads in all the directions [4]. However, it is worth mentioning a few drawbacks. In 3D reinforcements, the crimp due to the network of yarns is much more important than in $2 \mathrm{D}$ weaves and so is the damage due to the weaving and handling of yarns [5]. Furthermore, an intermediate fiber volume fraction

\footnotetext{
* Corresponding author. Tel.: +33 3277123 21; fax: +33 327712981 . E-mail address: philippe.le.grognec@mines-douai.fr (P. Le Grognec).
}

(40-50\%), due to the presence of unavoidable spaces between yarns is inherent to 3D structures, with a rare threshold of $60 \%$ [6].

Among 3D interlock structures, the layer-to-layer angleinterlock configuration provides the best answer to these mechanical requirements together with formability [3]. But very few industrial applications involving these types of reinforcement were attempted until now. The main reason is the present lack of knowledge about their mechanical behavior together with the difficulties encountered when dealing with their geometrical modeling. Their use just recently began to spread in aeronautics and aerospace structures (parts prone to impacts, such as rotor blades and landing gears). The analysis of ballistic protection for bullet-proof vests and vehicles is under consideration. Future applications could be front ends and leading edges of ships and boat hulls, sensitive to slamming. All these applications are revealing the potential of 3D interlock composites and show the growing interest of industrials for this type of materials.

The use of 3D composites requires a realistic geometrical and material representation in order to perform an accurate mechanical analysis. To do so, the study of the weaving and of the manufacturing process leads to a proper understanding of the final composite structure. During the weaving of a 3D fabric, a global distortion may occur because of its thickness, the residual stresses in the yarns and the interlacing [7]. Contrary to the single ply of a 2D woven fabric, the layers of a 3D fabric may translate horizontally due to the take-up motion (Fig. 1). Another modification is yarn crimp which is a local deformation of the yarn path, due to yarn interlacing and tension. Yarn crimp may be emphasized 


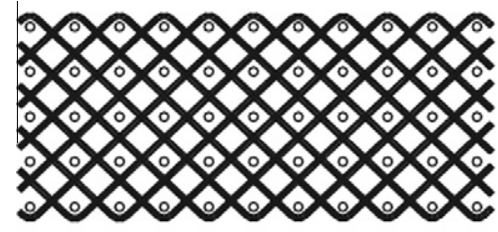

(a)

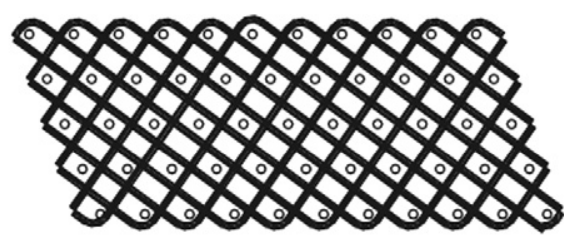

(b)

Fig. 1. Idealized architecture (a) and modified architecture after taking-up from the loom (b) [7].

during manufacturing processes (RTM, for example) due to compaction and nesting $[8,9]$. This phenomenon is already observed in laminate manufacturing [10] and may be more important in distorted yarns of 3D woven fabrics (Fig. 2).

All these modifications of the architecture (crimp, compaction, nesting), inherent to the manufacturing of 3D composites, lead to a decrease of the mechanical properties. Their consideration in a model is necessary to improve the prediction of the mechanical behavior.

As far as data are concerned, not only the geometry but also the material properties play a major role in the mechanical analysis of a textile reinforced composite. The influence of yarn damage due to the weaving is an important feature which should be taken into account to get closer to the true homogenized behavior of the composite material. That damage may be much more important in a 3D weaving than for a classical 2D one. Lee et al. studied the tow damage during the weaving of 3D carbon-fiber orthogonal fabrics [11]. It is due to abrasion and breaking of fibers when sliding in the loom. The authors compared single carbon composite yarns before and after weaving and pointed out a decrease of $12 \%$ of the mechanical strength. Archer et al. also studied the damage occurrence during the weaving of carbon-fiber angle-interlock fabrics [12]. The tensile strength of dry yarns from the fabric was found to be $9.4 \%$ lower than the nominal value before weaving. The importance of damage in this study will be discussed in more details in Sections 3.2 and 4.1.

The final architecture in the composite material is thus dependent of the parameters of the weaving and of the composite manufacturing process. It is essential to note that a complete mechanical study must be based on the whole manufacturing history. Most of the authors only consider one of the aspects of the manufacturing previously cited. As for example, some mechanical methods are listed below.

The classical laminate theory is the basis of 2D composite mechanics and may be used as a starting point in the assessment of the effective mechanical behavior of 3D interlock composites. Indeed, in a first approach, the interlock fabric could be considered as a laminate of 2D fabrics constituted of identical successive warp and weft layers with different orientations. That decomposition is clearly inappropriate here since it does not take into account the waviness of the woven yarns and the ply interconnectivity. Thus, the classical laminate theory cannot be used anymore in the case of interlocks or 3D fabrics in general.

Specific semi-analytical and numerical approaches have been proposed to study the mechanical behavior of 3D reinforced composites, as a replacement for the well-known laminate theory. The Orientation Averaging Model is an analytical approach developed by Cox et al. and based on a volume averaging of the straight yarn matrices [3]. The waviness is taken into account through rigidity loss factors for each type of yarn, known as knock-down factors.

Some approximate numerical averaging approaches have also been developed to assess the elastic properties of such composites using discretization methods such as the XYZ model [13], or using direct methods of 3D calculation on a modified structure with simpler material properties, such as the Binary Model [14]. In both models, the in-plane and through-the-thickness moduli are still overestimated, due to a coarse geometrical modeling (assembled micro-blocks for the XYZ model or yarns with 2-node elements for the Binary Model).

More recently, specific textile modeling softwares, such as Wisetex [15], TexGen [2] or TexEng, have been developed. They generally include both geometrical modeling (up to the filament scale) and mechanical simulation. For example, numerical results in Wisetex generally show a good consistency with experimental data but may often lead to discrepancies due to the limitations of the homogenization scheme employed.

In this work, the objective is to ease the mechanical study of a 3D interlock composite, using a high-performance tool. The final state of the interlock fabric in the composite depends on the manufacturing parameters (weaving and process), which may produce an architecture different from the original definition before weaving. Thus, on account of numerous existing geometries, a parameterized architecture is developed by means of a Python program, using Abaqus software in the context of the finite element method. Indeed, due to the strong heterogeneity and the complex architecture of such materials, a rigorous periodic homogenization scheme is (a)

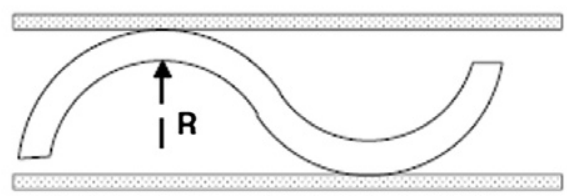

(b)

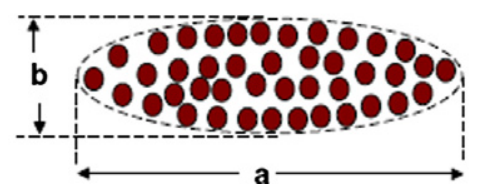



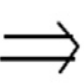

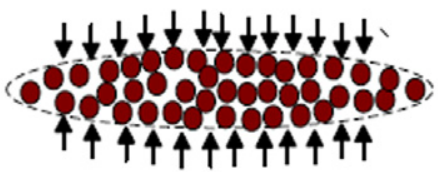

Fig. 2. Waviness change (a) and cross-section variation (b) due to compaction [10]. 
preferred here to assess the membrane and bending elastic behaviors of the equivalent shell-type structure, in the framework of the Love-Kirchhoff plate theory. Moreover, special attention is paid to the actual textile geometry and material parameters thanks to optical observations and measurements of mechanical properties of yarns extracted from woven fabrics. Hence, this paper proposes a general numerical tool facilitating the meso-scale geometrical modeling and the determination of the effective elastic properties of a 3D interlock composite. The numerical solutions are then compared to experimental tensile and bending results for validation.

\section{Composite materials reinforced with 3D interlock woven fabrics}

3D interlock structures are fabrics, which means that they are directly woven in the three dimensions with Z-yarns along the thickness linking different layers together. For that matter, Bogdanovich and Mohamed pointed out the difference between multilayer textiles which generate 3D structures thanks to the stacking of plies and fabrics like interlocks which are basically 3D [16]. Many types of architectures can be defined but there is no general data base for now. Boussu et al. recently suggested a unique parameterized architecture thanks to a complete inventory of the geometric parameters in order to improve the representation and classification of 3D interlock structures and better identify the adequate architecture relative to given mechanical requirements [17]. Indeed, all these parameters, among which the number of layers, the number of yarns by weave repeat and the binding depth, are essential in any geometrical and/or mechanical modeling. Three families can be distinguished among the 3D woven structures:

- the orthogonal interlock where the Z-yarns are perpendicular to the weaving plane and go through the whole thickness only between two columns of weft yarns;

- the through-thickness angle-interlock where the Z-yarns go through the whole thickness across more than two columns of weft yarns;

- the layer-to-layer angle-interlock where the Z-yarns link at least two plies.

The latter configuration seems to be more suitable to fulfill many mechanical requirements and formability ones. Moreover, as the orthogonal and through-thickness angle-interlock structures have been studied by many authors up to now $[4,6,13,14]$, the choice of the little-known layer-to-layer architecture has been retained in this study as it allows to investigate the effect of the orientation and waviness of binding yarns on the mechanical behavior of the composite material.

In the sequel, the architecture considered is an 8-layer reinforcement with carbon fibers. The repeated pattern is defined as follows: the weft binders link two successive plies together. The paths of the yarns and the section shape are defined using geometrical parameters, such as the fiber volume fraction, waviness, crimp and nesting of the yarns due to the weaving and composite process. A 3D realistic representation of the interlock architecture has been defined on the basis of optical observations and measurements achieved on cross-sections of the composite (Fig. 3).

3D interlock fabrics are usually woven on classical 2D looms [16]. The reinforced composite can then be manufactured by several Liquid Composite Molding (LCM) processes: RTM, VARTM or Infusion. The latter has been selected because of its growing popularity in the industry for the ease of preparation thanks to the lack of the upper part of the mould and the low-level deformation of the structure when submitted to moderate vacuum. This manufacturing process is discussed in more details in Section 3.3.

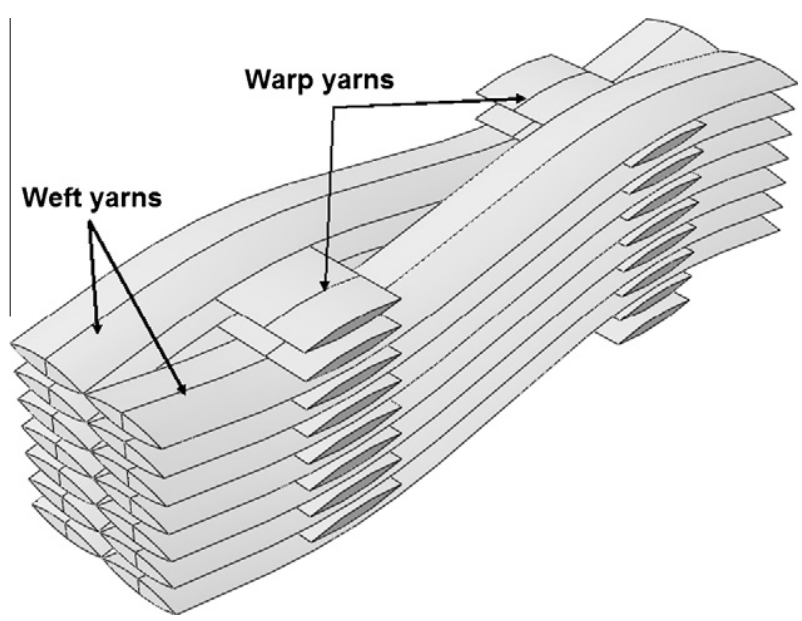

Fig. 3. Layer-to-layer angle-interlock architecture.

\section{Influence of manufacturing parameters on the definition of the 3D interlock composite}

An analysis of the weaving and infusion processes is required for a correct description of the geometry and material properties of the reinforced composite. First, the weaving itself causes a modification of the architecture compared to the theoretical one, and yarn damage. Then, the infusion process is also supposed to affect the final geometry of the reinforcement in the composite material due to transverse compaction.

\subsection{Effect of tensioning on the architecture during weaving}

All the fabrics used in this study are produced with the laboratory-scale ARM-Patronic loom, dedicated to the weaving of fabric samples (Fig. 4). The fibers are made of carbon HexTow ${ }^{\circledR}$ IM7 from Hexcel (Table 2).

The weaving on classical looms can be divided into the following steps: warp beam winding (or warp creel set-up), warp let-off, warp tensioning, shedding, weft insertion, beating, and taking-up of the finished fabric [18]. In this case, a few differences are noteworthy. There is no warp beam winding and let-off. The pristine bobbins are set up on a creel, the yarns pass through an alignment device and then through the eyes of the heedles. Thanks to the successive raising and lowering of the warp yarns, the weft yarns can be inserted and beaten. The advancement of the weaving is possible thanks to the translation of the set heedles/reed. The produced reinforcement has binding warp yarns and straight weft ones and exhibits some regularity problems. Thereby a tensioning system is added at the end of the loom in order to get a more regular and controlled fabric. But the respective roles of the yarns are reversed with this final tension: the warp yarns become straight whereas the weft ones turn out to be the linking yarns. This new architecture is then studied in the sequel. This reversal is noticed through the cross-section observations (Figs. 6 and 5) and it may change the material strength according to the direction. This geometrical change has been implemented in the proposed numerical tool in order to keep a wide range of interlock representations.

The effect of tensioning on the cross-section shape is also analyzed. The first interlock fabric displayed lenticular shape for both types of yarn. The tensioning creates two section shapes: lenticular for the warp yarns and trapezoidal for the weft ones. This change is also due to the beating, dependent of the manual action of the weaver. The straight warp yarns make up parallel channels where 


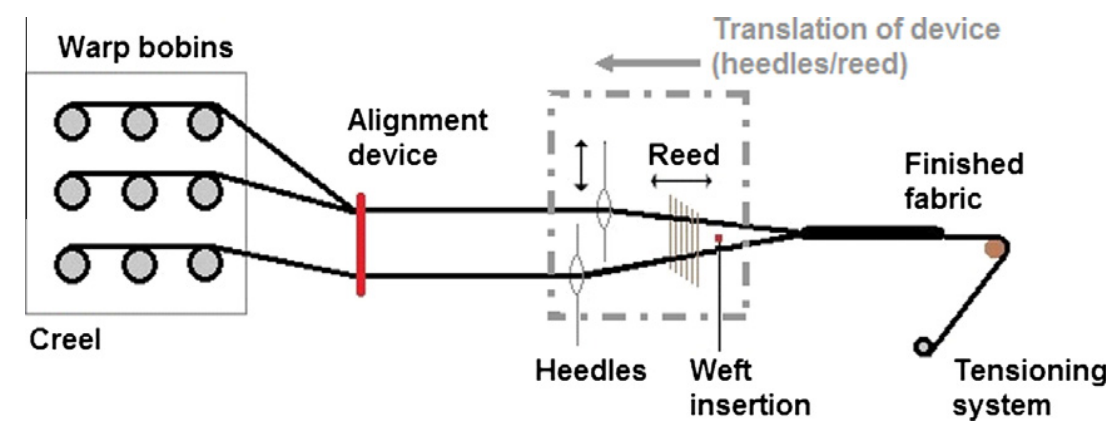

Fig. 4. Scheme of a weaving loom for 3D interlock fabric.

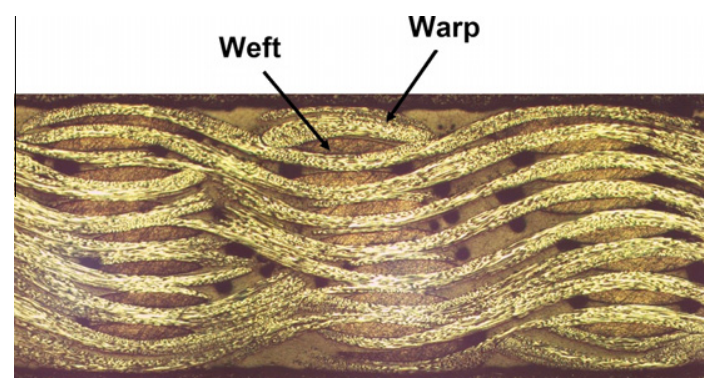

Fig. 5. Fabric woven without tensioning system - wavy warp yarns.

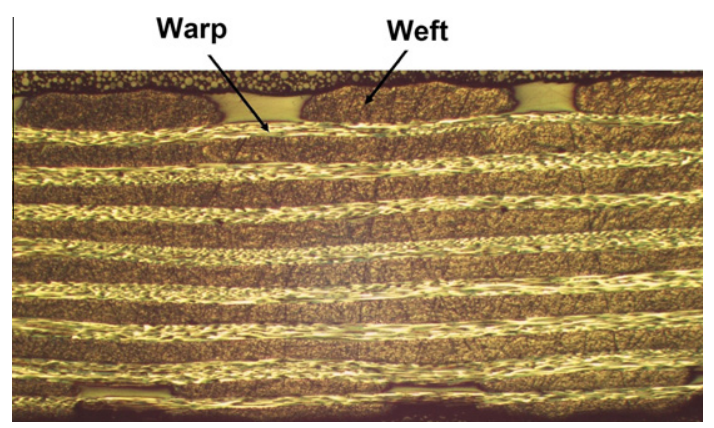

Fig. 6. Fabric woven with tensioning system - straight warp yarns.

the untightened weft yarns conform to each other with a trapezoidal section shape. This high density of weft yarns allows better mechanical properties in this direction. It is essential to keep in mind that the second architecture is a particular case, due to tension and beating, of a more general geometry with binding warp yarns and lenticular cross-sections for all yarns. The tensioning parameter allows to change the architecture without modifying the definition of the fabric. In the sequel, a lenticular section shape is attributed to all the yarns in order to keep the general purpose of this study.

\subsection{Effects of $3 D$ weaving on carbon tows}

Two aspects are studied in particular: the yarns passing into the loom and the waviness and crimp of the weft tows. The handcrafted weaving allows to be more careful with the monitoring and the change of broken yarns. Warp yarns can be damaged during the weaving, which in turn may influence the final strength of the material. The weft yarns are inserted manually and the operator is assisted for the warp yarns handling. Contrary to 2D fabrics, yarns are handled many times in 3D interlock fabrics - as many as the number of layers. Warp tows open a shed thanks to the ertical movements of the heedles in order to stack the weft yarns on the same vertical orientation.

As an illustration, in Fig. 7, the warp yarns are moved in order to open a path for the manual insertion of the weft yarn 1, and then replaced to lock in this yarn and open a path for the weft yarn 2 , and so on for the whole column of weft yarns. Those repeated yarn motions through the eyes of the heedles give rise to damage by friction (Fig. 8): in the eyes, against the heedles and against the other yarns (the wide warp tows are in contact). The warp filaments are weakened due to abrasion. The effect of the reed makes the damage deeper due to the friction against the warp yarns under tension. The weft tows undergo another effect of the loom. They are submitted in the rapier to multiple torsion efforts during the shedding.

As a consequence, the material properties of the yarns and therefore those of the manufactured fabric and composite decrease significantly.

The second influence is the waviness of the weft yarns. They are inserted in the shed and blocked by the warp tows. Then they become wavy as the warp ones are still straight. At the interlacing points between the warp and weft yarns, a flexural loading occurs in the latter. As the yarn is thin (6 k carbon yarn), the curvature radius is low, what may create stress concentration regions and so promote damage. Furthermore, during the beating, the reed packs two or three times the weft yarn, what might also increase the previous damage.

The recognition and the quantization of these phenomena are important in order to make the numerical model closer to the reality of the composite. Tensile tests are thus performed on single yarns before and after weaving to evaluate the stiffness decrease due to the weaving (results are presented in Section 4.1).

\subsection{Composite manufacturing process}

Composite samples are manufactured prior to characterization. The choice of infusion (vacuum pressure inferior to $0.1 \mathrm{MPa}$ ) was made among the different LCM processes available, as it is a simple closed-mould technique and induced a minimum of deformations.

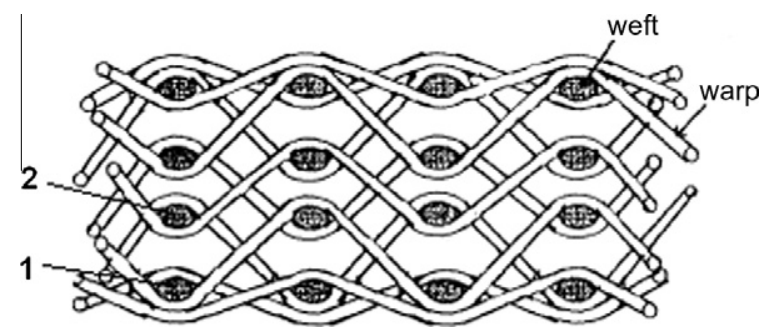

Fig. 7. Order of insertion of weft yarns [19]. 


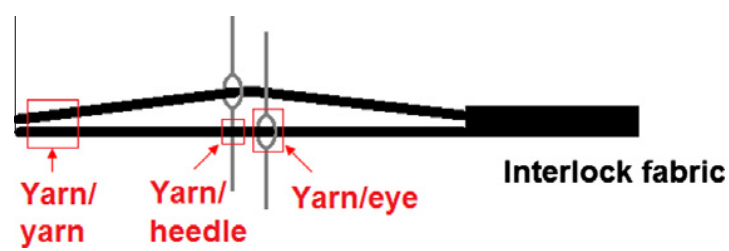

Fig. 8. Yarn damage in the raising/lowering device.

All the different plies are laid down on a flat mould, as shown in Fig. 9, and the vacuum bag is sealed. The resin is an Epolam 5015 epoxy, mixed with the 5016 hardener (Axson Technology, Table 1). Its viscosity at $25{ }^{\circ} \mathrm{C}$ is low (0.21 Pa.s) to ensure a good impregnation of the fabric. Then, the composite is post-cured during $2 \mathrm{~h}$ at $50{ }^{\circ} \mathrm{C}$ to complete the polymerization.

\subsection{Microscopic observations}

The aim of this subsection is to study the quality of the composite, estimated by the quantity of porosities and to obtain the yarn dimensions. Therefore, cross-sections of the 3D interlock composite are sampled in different locations and observed under an optical microscope. By definition, a porosity is a micro-cavity due to the trapping of air, gas or solvent during the impregnation stage. As the porosity scale of the fabric is double (filament and yarn scales), two types of porosity exist: the micro-porosity (between the fibers inside a tow) and the macro-porosity (between the yarns). The generation of porosities is a complex phenomenon which depends on the velocity and orientation of the flow front, the capillary pressure and the wettability of the fibers, among other things. The quantity of porosities defines the quality of the manufacturing which influences the mechanical behavior of the composite.

Cross-sections in the interlock composite plate are sampled in different locations, polished and then observed under a microscope equipped with a CCD camera. The porosity volume fraction is calculated from the measured porosity surfaces (Fig. 10). The size of every single porosity on each sample has been measured and its nature (micro- or macro-porosity) is recorded. It should be noted that Fig. 10 displays only a third of the actual sample size.

The global averaged porosity rate is low, around $0.6 \%$, with some samples showing maximal porosity levels of $2 \%$. It proves the good quality of the material. This very low rate also justifies a numerical study of the elastic mechanical behavior of the composite, without modeling the porosities and their inelastic behavior. The samples also show that the porosities are mostly located close to the interlace of the warp and weft yarns, as it is depicted in Fig. 10 and schematized in Fig. 11. Even though the porosity levels are low, the porosities may be strongly concentrated. Their presence between the warp and weft yarns can be the location of stress concentration and thus of damage initiation, such as a decohesion phenomenon. The yarn/yarn and yarn/matrix decohesion might be affected by these porosities.
Table 1

Properties of Epolam 5015 epoxy resin.

\begin{tabular}{ll} 
Maximum stress at failure & $105 \mathrm{MPa}$ \\
Young's modulus & $3 \mathrm{GPa}$ \\
Failure strain & $6 \%$ \\
Viscosity at $25{ }^{\circ} \mathrm{C}$ & 0.21 Pa.s \\
\hline
\end{tabular}

Table 2

Properties of HexTow ${ }^{\circledR}$ IM7 carbon fibers

$\begin{array}{ll}\text { Tensile strength } & 5310 \mathrm{MPa} \\ \text { Young's modulus } & 276 \mathrm{GPa} \\ \text { Failure strain } & 1.8 \% \\ \text { Fiber density } & 1.78 \\ \text { Number of filaments } & 6000 \\ \text { Fiber diameter } & 5.2 \mu \mathrm{m}\end{array}$

The other purpose of the microscopic observations is to measure the dimensions of the yarns in the composite. The shape of the cross-sections is found to be lenticular for the warp yarns and trapezoidal for the weft ones, as seen in Fig. 10. As mentioned in Section 3.1, a lenticular shape is set to all the cross-sections, in order to keep the general nature of the geometry. It can be noticed that the numerical lenticular weft section is equivalent to the real one in terms of surface area (Fig. 12). The spaces between yarns and the dimensions of the cross-sections (width and thickness) are measured accurately. The averaged dimensions are listed in Table 3.

It should also be mentioned that the cross-sections of the weft yarns are vertically misaligned due to the action of the reed during the weaving and the residual stresses in the yarns (Fig. 10). In this study, the porosities and the nesting are not considered at first to simplify the geometrical modeling but they could be implemented in a further simulation step.

Calcination tests are performed to measure the fiber fractions in the composite. It also allows to distinguish the fractions in both directions (Table 4). They can be then compared to fractions in the numerical model, in order to assess the accuracy of the modeling.

The fiber volume fractions inside the yarns are calculated from microscopic observations of cross-sections of binarized black and white pictures (Fig. 13). These fractions are used for the calculation of the moduli of the yarns in the numerical modeling (Table 5). The difference of fiber fractions in both directions is the consequence of a large number of weft yarns which creates a dense binding web and slightly compacts the warp yarns.

\section{Experimental results}

\subsection{Tensile tests on single yarn composite}

Some tests allow to assess the mechanical fiber properties, such as tensile tests on dry yarns or on dry yarns with impregnated end

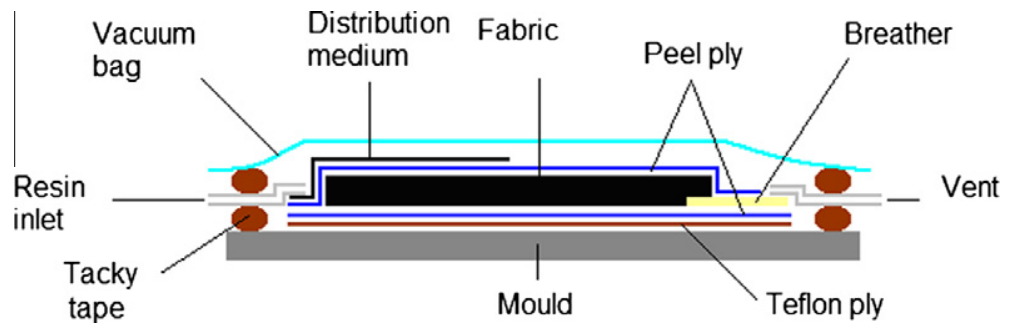

Fig. 9. Experimental device used for infusion. 


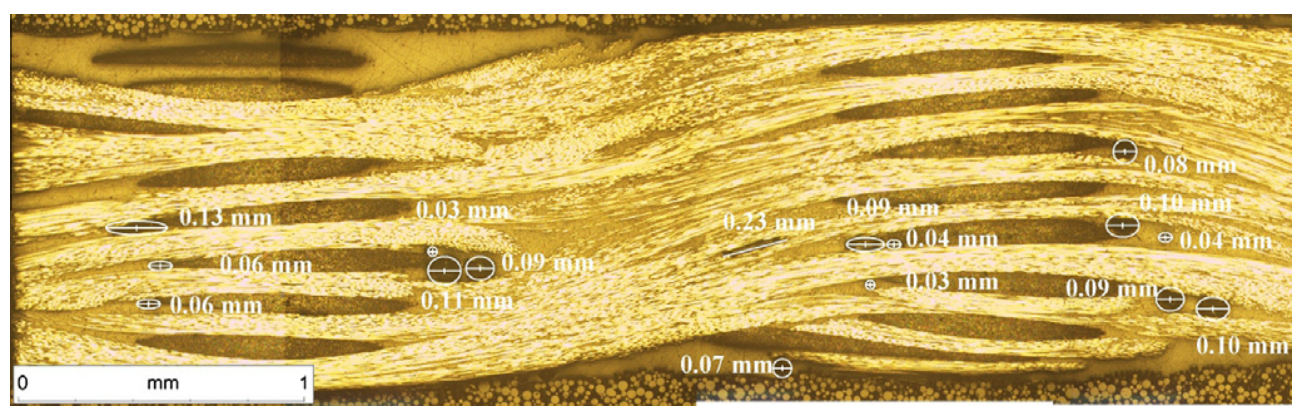

Fig. 10. Microscopic observation of cross-sections of the interlock composite in the weft direction.

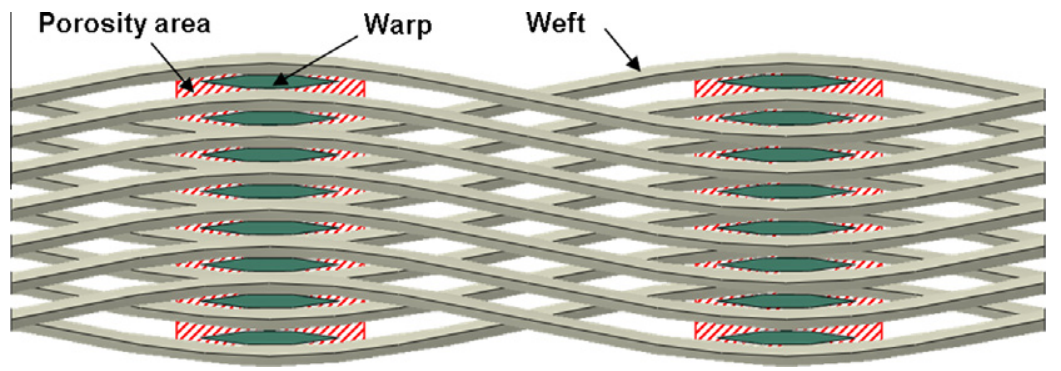

Fig. 11. Macro-porosities locations in the weft direction.

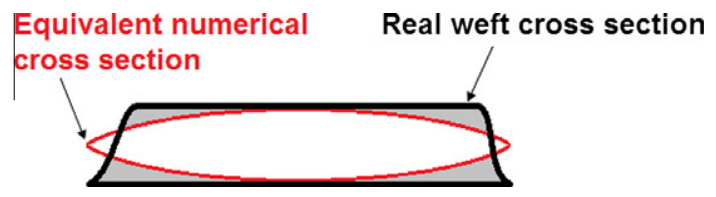

Fig. 12. Equivalent lenticular and real trapezoidal weft cross-sections.

Table 3

Geometric parameters of the 3D interlock composite.

\begin{tabular}{ll} 
Warp lens width & $1.313 \mathrm{~mm}$ \\
Warp lens thickness & $0.139 \mathrm{~mm}$ \\
Weft lens width & $1.152 \mathrm{~mm}$ \\
Weft lens thickness & $0.206 \mathrm{~mm}$ \\
Horizontal space between warp yarns & $3.554 \mathrm{~mm}$ \\
Horizontal space between weft yarns & $0.02 \mathrm{~mm}$ \\
Total thickness & $2.6 \mathrm{~mm}$ \\
\hline
\end{tabular}

tabs. The first type of specimen shows the difficulty of the tensile simultaneity on all the fibers thanks to a capstan. The second specimen implies stress concentrations at the interface between dry yarn and impregnated yarn, where rupture occurs without any sollicitation of the dry fibers. Thus, the ASTM D4018-99 standard method is followed to obtain the mechanical properties of the carbon fibers from tests on single composite yarns. Three types of sample are manufactured: one with yarns from the bobbin and two with woven yarns from the fabric in both directions. The tows are dipped into the resin and kept under tension during curing. Cardboard end tabs are then added onto the specimens to prevent from slipping in the jaws. The alignment of the yarns with the tensile direction and the jaw clamping effect (to avoid any slippage) were controlled with great attention. A series of five tests for each yarn type is performed on a 1474 Zwick machine with a $1 \mathrm{kN}$ force cell and the deformations are measured using an extensometer. As the fiber fraction is low in the yarns (15\%), the formulae from the standard for the tensile modulus of the fibers is changed with
Table 4

Fiber volume fractions in the whole composite and split into the warp and weft directions.

\begin{tabular}{llll}
\hline & Composite & Warp yarns & Weft yarns \\
\hline$V_{f}$ & $42 \%$ & $7 \%$ & $35 \%$ \\
\hline
\end{tabular}

the addition of a term accounting for the matrix, issued from the mixture law:

$E_{L}^{f}=\frac{\Delta P \times \rho_{f}}{\Delta \epsilon \times M U L}-\frac{\left(1-V_{f}\right)}{V_{f}} \times E^{m}$

where $\Delta P$ is the load variation, $\Delta \epsilon$ the strain variation, $\rho_{f}$ the fiber density, MUL the mass by unit length, $V_{f}$ the fiber fraction of the single composite yarn and $E^{m}$ the matrix modulus.

Table 6 displays the Young's moduli for all the types of yarn. The moduli of fibers from the bobbin and of warp yarns are very close to each other ( $1 \%$ of difference). There is no damage of the warp yarns during the weaving, despite of what was expected. It might be due to the low number of warp yarns which are thus not in close contact to each other and against the heedles thanks to the alignment device which allows the differentiation of the warp yarns. Conversely, a loss of about $8 \%$ of the longitudinal modulus is noticed for the weft tows, due to beating and waviness as mentioned in Section 3.2. The low dispersion of the results proves their reliability.

Values in Table 6 will be used as input data in Section 5, instead of the values simply deriving from the classical mixture law using the fiber manufacturer moduli.

\subsection{Tensile and flexural tests on $3 D$ interlock composite samples}

Experimental tests on composite samples cut out from the infused composite plate have been conducted in order to validate numerical results.

The monotonous static tensile tests are performed on an 8-layer interlock composite according to the ISO 527-4 standard. Rectangular specimens without end tabs $\left(250 \times 25 \mathrm{~mm}^{2}\right)$ are tested on a 1474 Zwick machine with a $100 \mathrm{kN}$ force cell. Strains are mea- 


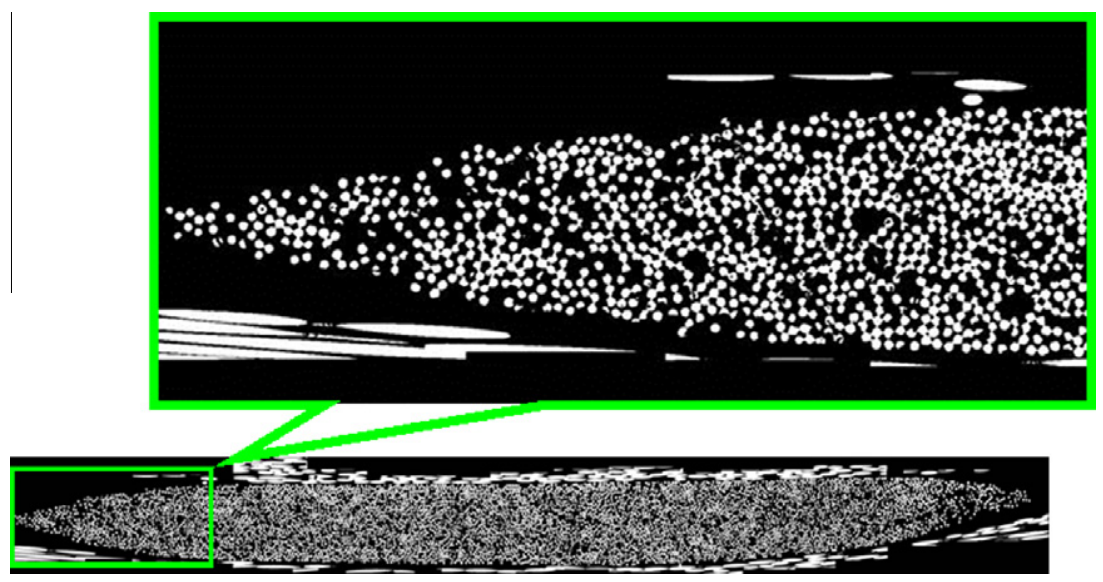

Fig. 13. Microscopic transversal cross-section of a warp yarn.

Table 5

Fiber volume fractions in both types of yarn.

\begin{tabular}{lll}
\hline & Warp yarn & Weft yarn \\
\hline$V_{f}^{\text {yarn }}$ & $65 \%$ & $60 \%$ \\
\hline
\end{tabular}

Table 6

Mechanical properties of yarns before and after weaving.

\begin{tabular}{ll}
\hline & Young's modulus \\
\hline Yarn from the bobbin & $289 \mathrm{GPa}( \pm 6 \%)$ \\
Warp yarn from the fabric & $287 \mathrm{GPa}( \pm 2 \%)$ \\
Weft yarn from the fabric & $264 \mathrm{GPa}( \pm 6 \%)$ \\
\hline
\end{tabular}

Table 7

Elastic tensile and flexural moduli of the interlock composite.

\begin{tabular}{lll}
\hline & Tensile moduli & Flexural moduli \\
\hline Warp (1) & $E_{1}^{m}=12.3 \mathrm{GPa}( \pm 5 \%)$ & $E_{1}^{f}=17.3 \mathrm{GPa}( \pm 10 \%)$ \\
Binding weft (2) & $E_{2}^{m}=64.5 \mathrm{GPa}( \pm 7 \%)$ & $E_{2}^{f}=58.1 \mathrm{GPa}( \pm 4 \%)$ \\
\hline
\end{tabular}

sured using an extensometer. The flexural tests are performed according to the ISO 14125 standard. The rectangular specimens $\left(100 \times 15 \mathrm{~mm}^{2}\right)$ are loaded with a constant speed of $4 \mathrm{~mm} / \mathrm{min}$. The equivalent tensile $(\mathrm{m})$ and 3-point flexural $(f)$ moduli of the 8 -layer interlock composite in the warp (1) and weft (2) directions are averaged each from a series of five tests (Table 7). These experimental results will be compared to numerical ones in Section 6.

\section{Numerical prediction of the equivalent mechanical behavior}

Full 3D finite element simulations of 3D interlock composites is a computationally intensive task due to the strong heterogeneity of such structures. An alternative consists in replacing the 3D heterogeneous material by an equivalent homogeneous plate suitable for structural applications. As the interlock geometry is periodic in the fabric plane (in both directions), the calculation of the effective properties can be performed using an homogenization procedure on a periodic unit cell. Numerical finite element computations on an elementary volume involving different simple loadings will enable to derive the macroscopic elastic in-plane moduli (or stiffnesses). The periodic homogenization technique is briefly presented below and then applied to a periodic unit cell of the 3D interlock composite.

\subsection{Periodic homogenization}

The approach described in this work has already been applied by Buannic et al., among others, in the framework of sandwich structures [20]. Dealing with asymptotic homogenization, two scale parameters are introduced in the formulation of the 3D problem of a thin periodic structure: $e$, the thinness of the plate and $l$, the periodicity of the plate, which are supposed to be of the same order of magnitude. Then an asymptotic expansion method can be used with only one parameter. Two coordinate systems $y_{i}$ $(i=1,2,3)$ and $x_{\alpha}(\alpha=1,2)$ are defined (with subscript 3 for the thickness direction), corresponding to the microscopic and macroscopic scales, respectively. Next, the displacement field solution of the $3 \mathrm{D}$ elasticity problem is searched under the following form (with $y_{\alpha}=x_{\alpha} / e(\alpha=1,2)$ ):

$$
\begin{aligned}
\mathbf{u}(\mathbf{x})= & u^{(0)}\left(x_{1}, x_{2}\right)+e u^{(1)}\left(x_{1}, x_{2}, y_{1}, y_{2}, y_{3}\right) \\
& +e^{2} u^{(2)}\left(x_{1}, x_{2}, y_{1}, y_{2}, y_{3}\right)+\cdots
\end{aligned}
$$

The 3D problem is thus decomposed in a sequence of 3D microscopic and 2D macroscopic sub-problems, derived at each order of the infinitesimal parameter in the asymptotic expansion. Solving the 0th-order microscopic problem leads to the homogenized constitutive relations in the framework of the Love-Kirchhoff plate theory, as explained in the sequel.

The local strain field in the unit cell is additively decomposed into the macroscopic strain $\mathbf{E}+y_{3} \mathbf{K}$ (where $\mathbf{E}$ represents the membrane macrodeformation and $\mathbf{K}$ the curvature one) and a perturbation $\epsilon^{*}$ which accounts for the effect of the heterogeneities. This fluctuation of the strain tensor derives from a periodic displacement vector $\mathbf{u}^{\text {per }}$ and displays a null average value in the cell volume. The following elasticity problem can then be solved, with any enforced macroscopic strain field, and the displacement $\mathbf{u}^{\text {per }}$, the microscopic strains $\epsilon$ and stresses $\sigma$ as unknowns:

$$
\left\{\begin{array}{l}
\operatorname{div}(\boldsymbol{\sigma})=\mathbf{0} \\
\boldsymbol{\sigma}=\mathbf{C}: \boldsymbol{\epsilon} \\
\epsilon_{i j}=E_{i j}+y_{3} K_{i j}+\epsilon_{i j}^{*}\left(\mathbf{u}^{\text {per }}\right) \quad \text { with } i, j=1,2 \\
\boldsymbol{\sigma} \cdot \boldsymbol{n}=\mathbf{0} \text { on } \partial \Omega_{3} \\
\mathbf{u}^{\text {per }} y_{1}-\text { and } y_{2}-\text { periodic } \\
\boldsymbol{\sigma} \cdot \boldsymbol{n} \text { anti-periodic }
\end{array}\right.
$$

where $\mathbf{C}$ represents the local fourth-order elasticity tensor (which depends on the material at the local scale) and $\partial \Omega_{i}$ stands for the boundary surfaces of the unit cell with normal $\pm \mathbf{y}_{i}$. 
The macroscopic constitutive relations of the plate equivalent problem are classically written using the macroscopic in-plane stress resultants $\{N\}$ and moments $\{M\}$, which are simply derived from the average stresses by integration over the thickness. The homogenized constitutive equation can then be written as follows, in matrix notation:

$$
\left\{\begin{array}{l}
\{N\} \\
\{M\}
\end{array}\right\}=\left[\begin{array}{cc}
{[A]} & {[B]} \\
{[B]^{T}} & {[D]}
\end{array}\right]\left\{\begin{array}{l}
\{E\} \\
\{K\}
\end{array}\right\}
$$

where $[A],[B]$ and $[D]$ represent the effective stiffness matrices of the tensile, coupled and bending behaviors, respectively and $\{E\}^{T}=$ $\left\{E_{11}, E_{22}, 2 E_{12}\right\},\{K\}^{T}=\left\{K_{11}, K_{22}, 2 K_{12}\right\}$, in the frame of the LoveKirchhoff plate theory.

\subsection{Solution procedure}

The constitutive matrix in Eq. (4) is naturally symmetric so that only 21 components have to be determined. The 21 macroscopic strain fields applied on the heterogeneous unit cell are defined on an homogeneous cell, with the same mesh as the one used for the heterogeneous cell. The homogeneous cell is isotropic and ruled by the Hooke's law with the Lamé coefficients $(\lambda, \mu)$ :

$$
\begin{aligned}
& \boldsymbol{\sigma}=\lambda \operatorname{tr}(\boldsymbol{\epsilon}) \boldsymbol{I}+2 \mu \boldsymbol{\epsilon} \quad \text { with } \lambda=\frac{v E}{(1+v)(1-2 v)} \quad \text { and } \\
& \mu=\frac{E}{2(1+v)}
\end{aligned}
$$

where $\boldsymbol{\sigma}$ and $\boldsymbol{\epsilon}$ are the stress and strain tensors, $E$ and $v$ the Young's modulus and Poisson coefficient of the isotropic homogeneous material, and $\boldsymbol{I}$ the identity tensor. The volume $\boldsymbol{f}$ and surface $\boldsymbol{N}$ loadings are deduced from the local equilibrium equations, defined with boundary conditions on the lateral faces of the cell:

$$
\left\{\begin{array}{l}
\operatorname{div}(\boldsymbol{\sigma})+\boldsymbol{f}=\mathbf{0} \\
\boldsymbol{\sigma} \cdot \boldsymbol{n}=\boldsymbol{N}
\end{array}\right.
$$

where $\boldsymbol{n}$ is the outward normal unit vector from a given face. These boundary conditions are applied on the unit cell to create the different strain fields. Figs. 14 and 15 show the six elementary strain fields in membrane and curvature, respectively.

As a consequence, the 21 successive macroscopic strains are applied as initial deformation states on the heterogeneous unit cell, each representing one of the membrane or curvature macrodeformations or a linear combination of them, and the equilibrium state is reached under periodic boundary conditions. In each loading case, the strain energy $W$ is deduced and employed for the determination of the constitutive moduli, through the following relation:

$$
\begin{aligned}
W & =\frac{1}{2} \int_{\Omega} \boldsymbol{\sigma}: \boldsymbol{\epsilon} d \Omega \\
& =\frac{S}{2}\left(\{E\}^{T} \cdot[A] \cdot\{E\}+\{K\}^{T} \cdot[D] \cdot\{K\}+2\{E\}^{T} \cdot[B] \cdot\{K\}\right)
\end{aligned}
$$

where $S$ is the cell surface area in the reference plane.

\subsection{Finite element simulation}

\subsubsection{Geometrical modeling and material properties}

The finite element computations are performed on Abaqus software, using a home-made script Python program so as to automate the modeling procedure and the post-processing, namely the calculation of the effective constitutive matrix. The reinforcement considered features seven weft layers and eight warp layers (Fig. 3). The vertical space between yarns is null.
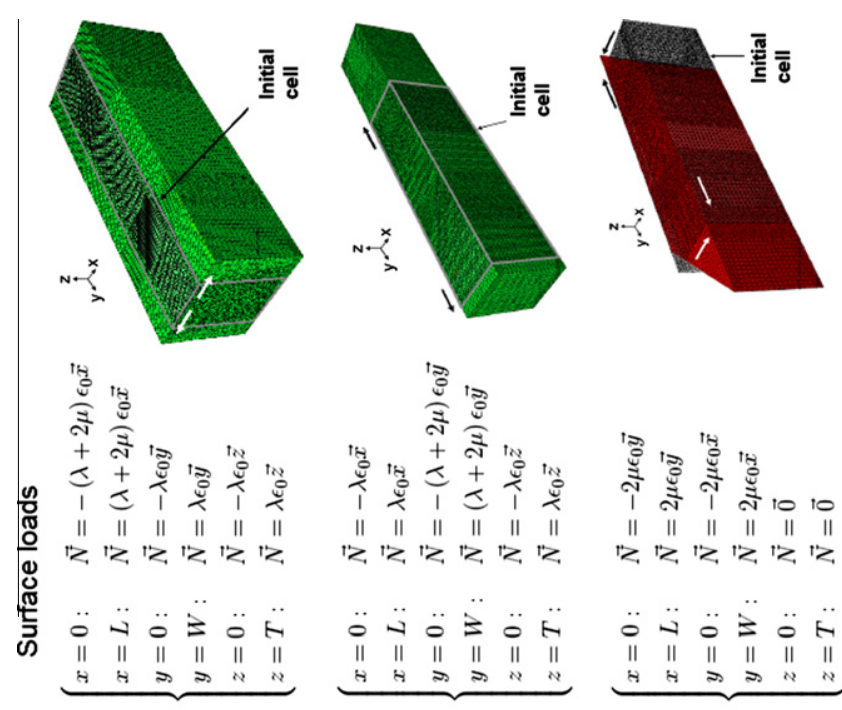

$1>1>1>1>1>$
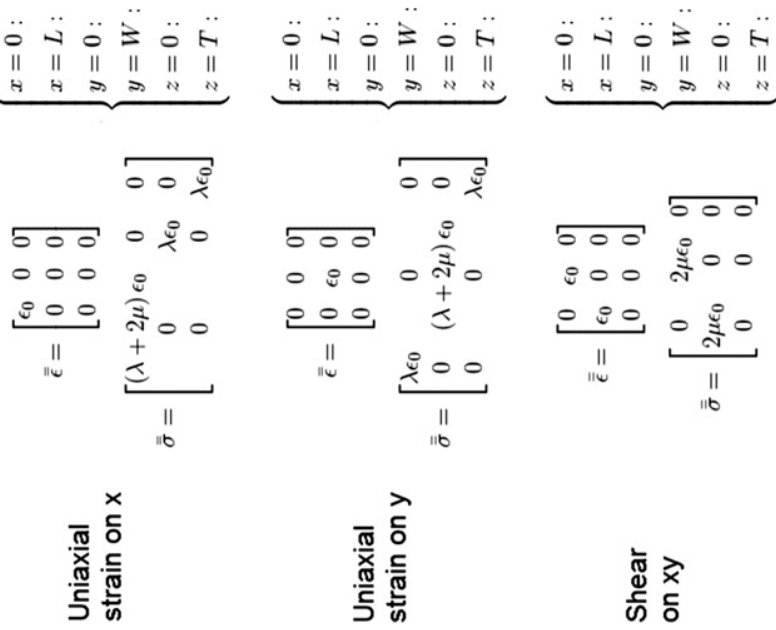

Fig. 14. Tensors, applied loads and strain fields in membrane.

The use of the described homogenization method makes necessary the periodic modeling of the interlock composite. However, this periodicity cannot be respected because of some previously noticed factors such as nesting, inhomogeneous yarns, yarn crimp and porosity. Thus, the geometry is idealized until then, assuming the absence of these factors. The modeling might not be representative of the real material due to these imperfections, which can be analyzed statistically and implemented. It is a subtle balance between the needs of a method and the representation of the material.

The unit cell is chosen as the smallest volume representing the whole structure by periodicity. The geometrical modeling of the unit cell is straightforward thanks to modifiable input parameters such as the dimensions of the yarns, horizontal spaces between yarns, and the mesh size.

As mentioned above, the longitudinal Young's moduli of impregnated yarns are simply derived from the mixture law, using the experimental values of fiber moduli found in Section 4.1 (Table 6) and fiber volume fractions in each yarn type (Table 5). The effective modulus of an undamaged tow $(187 \mathrm{GPa})$ is assigned to the warp tows whereas the binding weft tows are considered as damaged with a longitudinal modulus of $173 \mathrm{GPa}$. The other moduli are calculated from the HTA carbon fiber properties used by Cox et al. [3], which are supposed equivalent to these of IM7 carbon fibers (Table 8 where $L$ represents the fiber direction and $T, T^{\prime}$ the transverse directions of a yarn).

\subsubsection{Numerical results}

The above-mentioned general procedure leads to the complete stiffness matrix: 


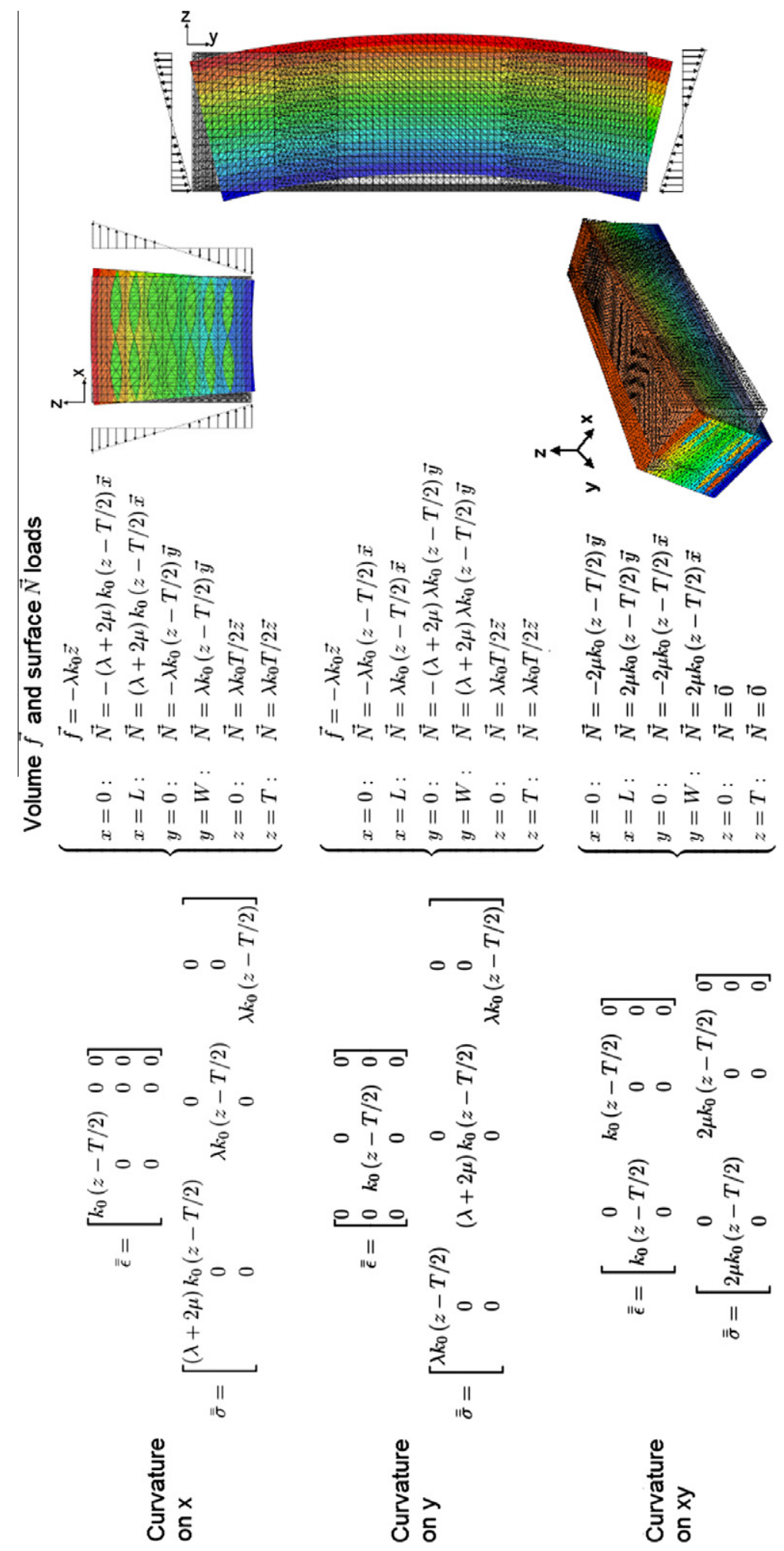

Fig. 15. Tensors, applied loads and strain fields in curvature.

$$
[C]=\left[\begin{array}{cccccc}
51,386 & 3113 & 3.9 & -11,700 & -3362 & 3.4 \\
3113 & 151,925 & 56 & -8638 & -34,406 & -14 \\
3.9 & 56 & 6493 & -3.7 & -12 & -1118 \\
-11,700 & -8638 & -3.7 & 35,751 & 4858 & -18.4 \\
-3362 & -34,406 & -12 & 4858 & 87,346 & -27.6 \\
3.4 & -14 & -1118 & -18.4 & -27.6 & 4333
\end{array}\right]
$$

It can be noticed that the components of sub-matrix $[B]$ as well as the terms $A_{13}, A_{23}, D_{13}$ and $D_{23}$ are very low in comparison with the others, excepted for $B_{11}$ and $B_{22}$ (Eq. (4)). The assumption of the null $B_{i j}$ terms leads to the decoupling between the membrane and flexural behaviors. The case of non null $B_{11}$ and $B_{22}$ might be due to the volume unbalance introduced by the new architecture. In the case of the former interlock (binding warp and straight weft), the $B_{i j}$ terms were null, what might be due to its balanced architecture 
(equivalent fiber volume fractions in both directions). Furthermore, the effective moduli calculated with null $B_{i j}$ terms are the same values as those calculated with the above sub-matrix $[B]$. Then, from the remaining terms, one can deduce the following in-plane elastic moduli, corresponding to the membrane $(m)$ and flexural $(f)$ behaviors, respectively:

$$
\begin{array}{ll}
E_{1}^{m}=17,303 \mathrm{MPa} & E_{1}^{f}=16,318 \mathrm{MPa} \\
E_{2}^{m}=51,116 \mathrm{MPa} & E_{2}^{f}=39,867 \mathrm{MPa} \\
G_{12}^{m}=2189 \mathrm{MPa} & G_{12}^{f}=1993 \mathrm{MPa} \\
v_{12}^{m}=0.0205 & v_{12}^{f}=0.0556 \\
v_{21}^{m}=0.0606 & v_{21}^{f}=0.135
\end{array}
$$

where 1 stands for the warp direction and 2 for the weft one.

\section{Validation and analysis}

In this section, a comparison between the deduced 2D equivalent behavior and the 3D heterogeneous plate is performed in membrane and flexural modes, in both directions. Then, a second comparison is performed with experimental tensile and bending tests.

The 3D heterogeneous plate is created by assembling unit cells together, only in the loading direction. The numerical simulation of the plate is restricted by the number of elements and so the number of merged unit cells. Simulations are achieved with one, two and four unit cells and the corresponding deformed shapes are compared with the analytical solutions based on the $2 \mathrm{D}$ equivalent properties.

In the weft direction, for a same tensile load, Fig. 16 shows that an improvement of the accordance between the $3 \mathrm{D}$ and $2 \mathrm{D}$ equivalent plates is achieved with an increasing number of cells (the maximal error at the plate end is $43 \%, 35 \%$ and $21 \%$, respectively for a 1-, 2- and 4-cell structure). In the warp direction, adding one cell decreases significantly the error from $73 \%$ (Fig. 17) to 13\% (Fig. 18) at the end of the structure. These results show the importance to use structures with large dimensions, to keep the scale separation effective (e.g. $l \ll 1$, as defined in Section 5.1). The local increase of 3D deformed shapes at the structure end is due to the side effects in the matrix.

In pure bending, the plates are composed of two cells in both directions and a moment is applied at the end of the structure. There is a good agreement in the weft and warp directions between the two models, with only $11 \%$ (Fig. 19) and $22 \%$ of error (Fig. 20), respectively. In the warp direction, the error might be further decreased with the add of cells in order to reach a longer structure as in the weft direction.

In the simple bending case with two cells, a difference of $22 \%$ is obtained in the weft direction (Fig. 21) whereas $60 \%$ of error is obtained in the warp direction (Fig. 22). The transverse shear effects are not considered in the Love-Kirchhoff theory, which may explain the discrepancies, but this feature can be introduced using higher-order terms in the asymptotic expansion to get better predictions.

Table 8

Mechanical properties of constituents and impregnated yarns.

\begin{tabular}{lllllll}
\hline & IM7 carbon fiber & \multicolumn{5}{l}{ HTA carbon fiber [3] } \\
\cline { 3 - 7 } & $E_{L}$ & $E_{T}, E_{T}^{\prime}$ & $G_{L T}, G_{L T}^{\prime}$ & $G_{T T}^{\prime}$ & $v_{L T}, v_{L T}^{\prime}$ & $v_{T T}^{\prime}$ \\
\hline Fiber & $287 / 264 \mathrm{GPa}$ & $17 \mathrm{GPa}$ & $55 \mathrm{GPa}$ & $6.7 \mathrm{GPa}$ & 0.25 & 0.27 \\
Resin & $3 \mathrm{GPa}$ & - & $1.1 \mathrm{GPa}$ & - & 0.35 & - \\
Yarn & $187 / 173 \mathrm{GPa}$ & $8.4 \mathrm{GPa}$ & $4.8 \mathrm{GPa}$ & $2.9 \mathrm{GPa}$ & 0.28 & 0.44 \\
\hline
\end{tabular}

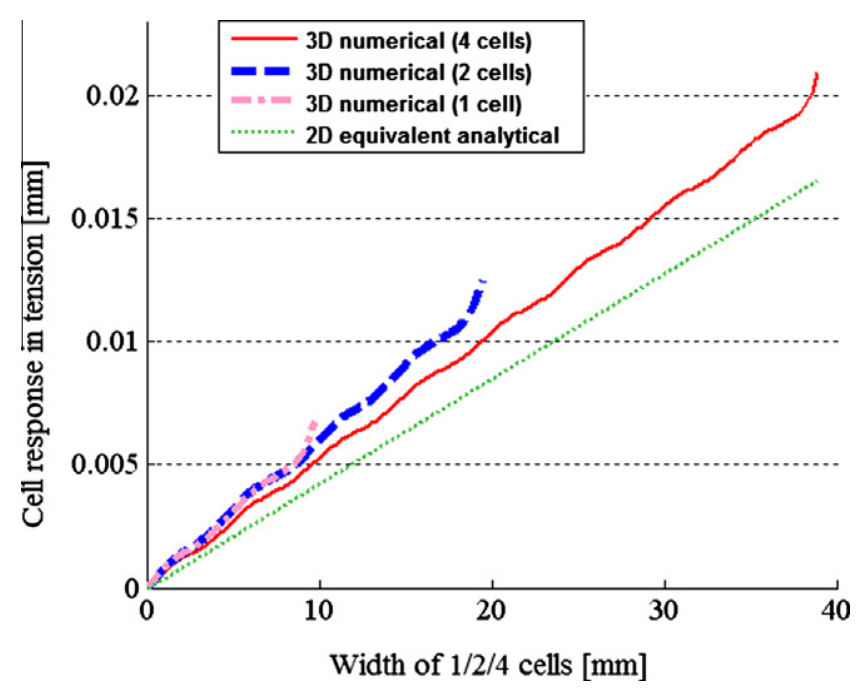

Fig. 16. Comparison between 3D numerical and 2D equivalent analytical responses in tension of $1 / 2 / 4$ cells in the weft direction.

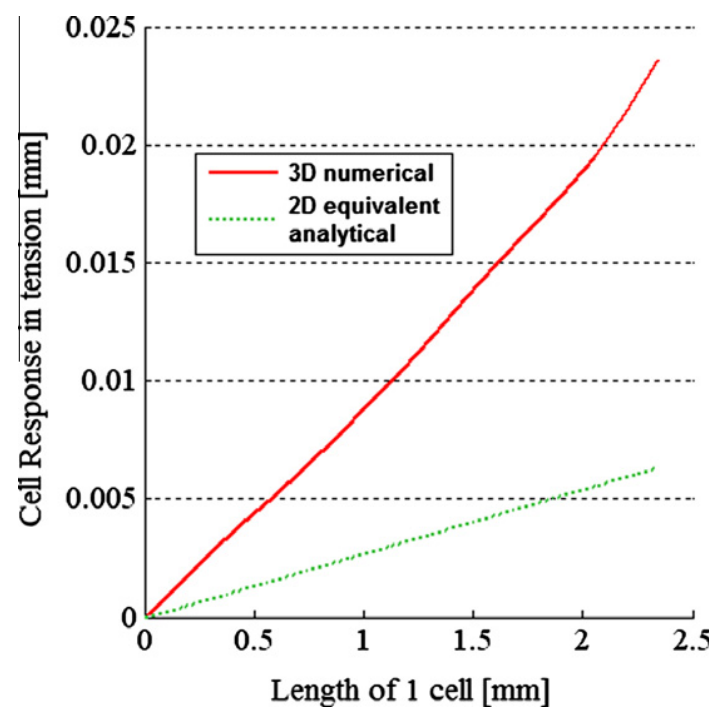

Fig. 17. Comparison between 3D numerical and 2D equivalent analytical responses in tension of one cell in the warp direction.

The equivalent elastic properties are already in good agreement with the 3D simulations in both tensile and pure bending cases, for a limited number of cells. A higher number of assembled cells would be required to really conclude about the accuracy of the results. However, this work was not achieved due to the limitation in computation resources.

2D equivalent results are then compared with experimental tensile and 3-point bending tests in the warp and weft directions, in the form of stress-strain and force-displacement curves (Figs. 23 and 24).

In the tensile case, the weft and warp averaged experimental curves show a non-linear behavior likely due to the damage initiation: yarn/matrix decohesion and friction. In the bending test, the composite material has a pure elastic behavior until rupture. The experimental and equivalent elastic moduli are compared in Table 9. Correlation is good in bending in the warp direction but could be improved in tension with a more precise modeling of the yarns (compaction and cross-section variation). In the weft direction, in both types of loading, the numerical moduli underes- 


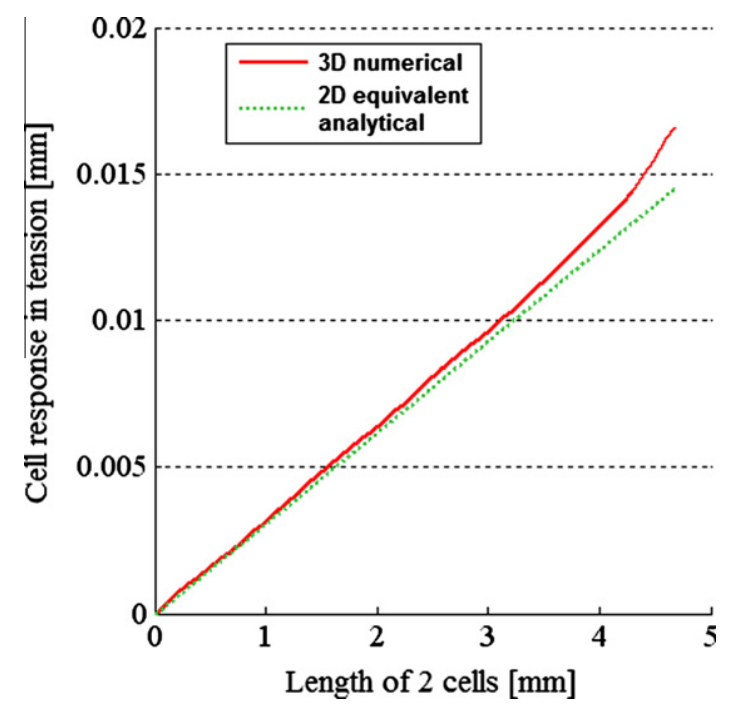

Fig. 18. Comparison between 3D numerical and 2D equivalent analytical responses in tension of two cells in the warp direction.

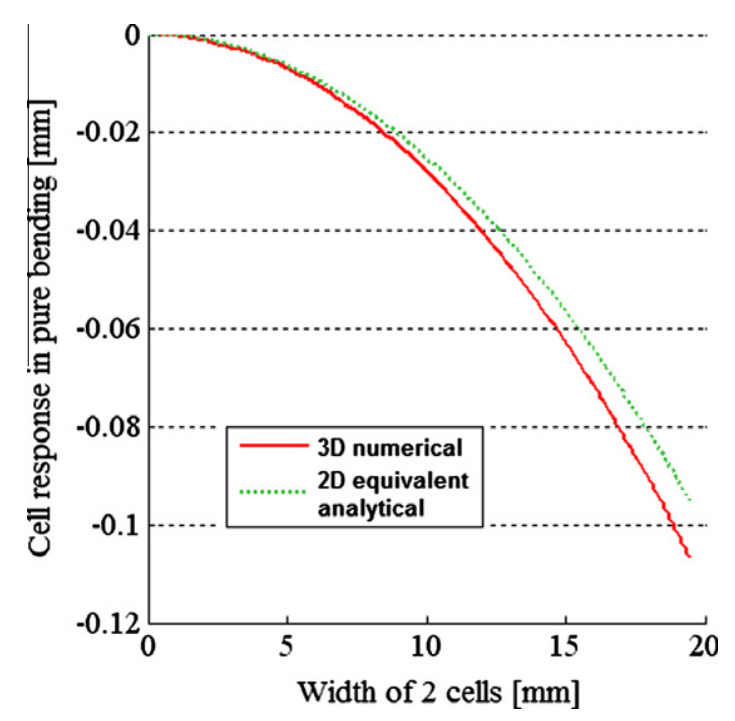

Fig. 19. Comparison between $3 \mathrm{D}$ numerical and $2 \mathrm{D}$ equivalent analytical responses in pure bending of two cells in the weft direction.

timate the experimental ones. These differences are mainly due to the numerical representation of the fiber volume fractions. Indeed, the numerical fiber volume fractions are lower of $4.4 \%$ in warp and $19 \%$ in weft direction than the measured ones in the composite (Table 4). In order to increase these values in the model, without changing the yarn dimensions, it is necessary to consider the nesting and thus a more densely packed architecture.

Furthermore, for comparison purposes, the predictions with all yarns considered as undamaged (namely a longitudinal modulus of $187 \mathrm{GPa}$ ) are plotted in the tensile and bending cases of the weft direction (Weft num 2D (undamaged yarns) curves). The average moduli would then be $55 \mathrm{GPa}$ (tensile) and $42.7 \mathrm{GPa}$ (bending): the difference of $7 \%$ with the first damaged equivalent moduli shows the importance of taking into account the real material properties of the yarns. The latter moduli with undamaged yarns seem to improve the agreement with the experimental results. However, as mentioned above, the improvement of geometrical modeling thanks to nesting will increase the equivalent moduli

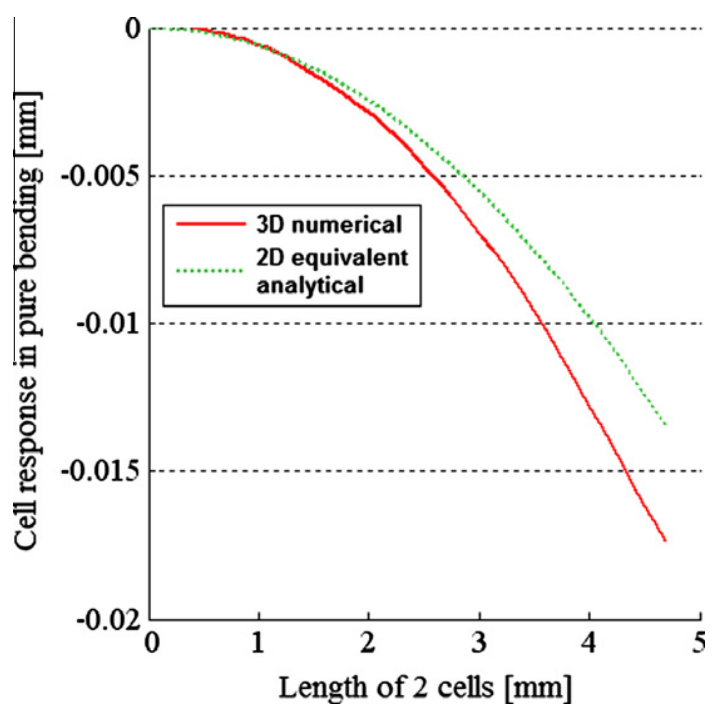

Fig. 20. Comparison between 3D numerical and 2D equivalent analytical responses in pure bending of two cells in the warp direction.

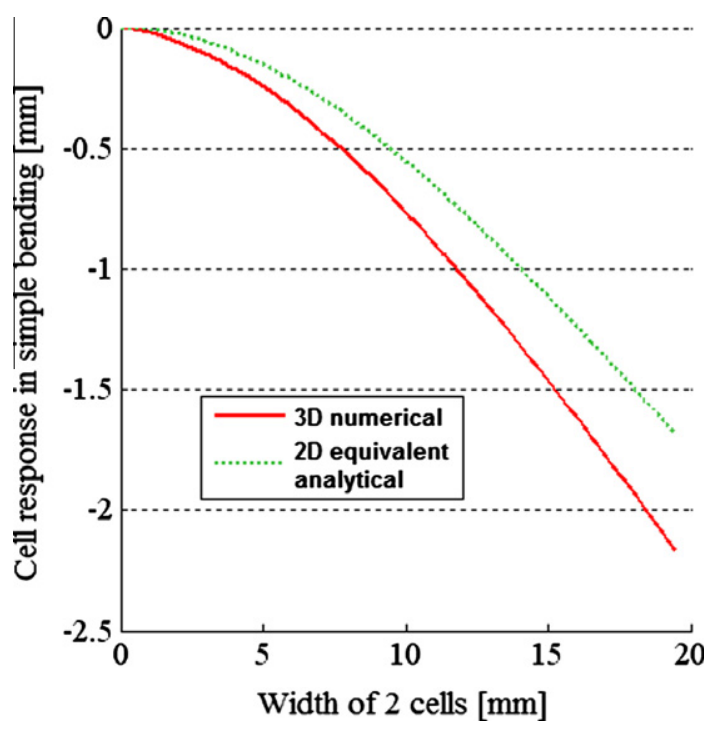

Fig. 21. Comparison between 3D numerical and $2 \mathrm{D}$ equivalent analytical responses in simple bending of two cells in the weft direction.

(with damaged/undamaged yarns or totally undamaged yarns) which will then overestimate the experimental values.

\section{Conclusion}

3D interlock composites make them candidates for specific applications thanks to their delamination and impact resistance. Their mechanical study needs new approaches different from the ones based on the laminate theory. In this work a numerical tool has been developed for the mechanical study of a carbon/epoxy layer-to-layer angle-interlock composite. The realistic modeling of the material in terms of geometry and constituents properties is essential for an accurate prediction of the mechanical behavior.

An analysis of the whole manufacturing of the composite highlights how the weaving and infusion process parameters can influence the final architecture. Some of these parameters such as dimensions, fiber fractions, yarn damage during weaving, are implemented into the automatic and parameterized modeling of 


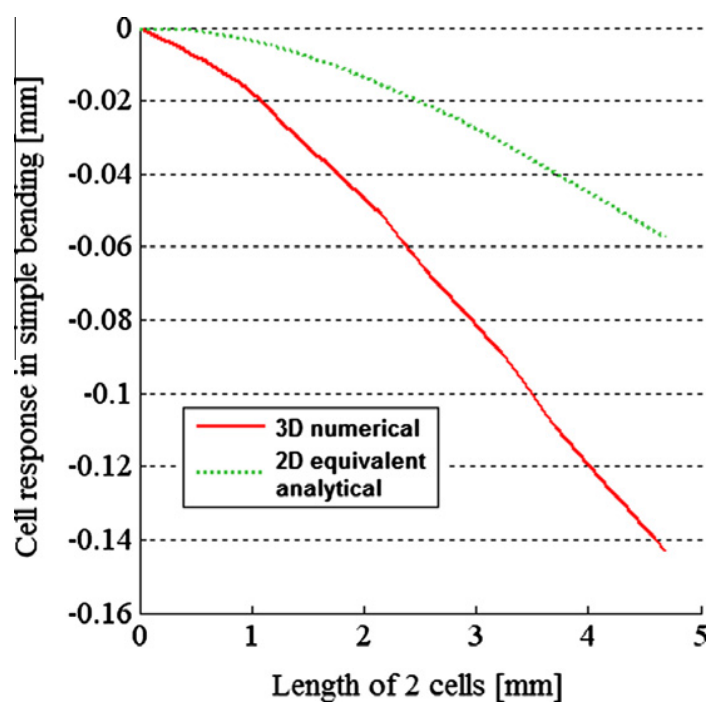

Fig. 22. Comparison between 3D numerical and 2D equivalent analytical responses in simple bending of two cells in the warp direction.

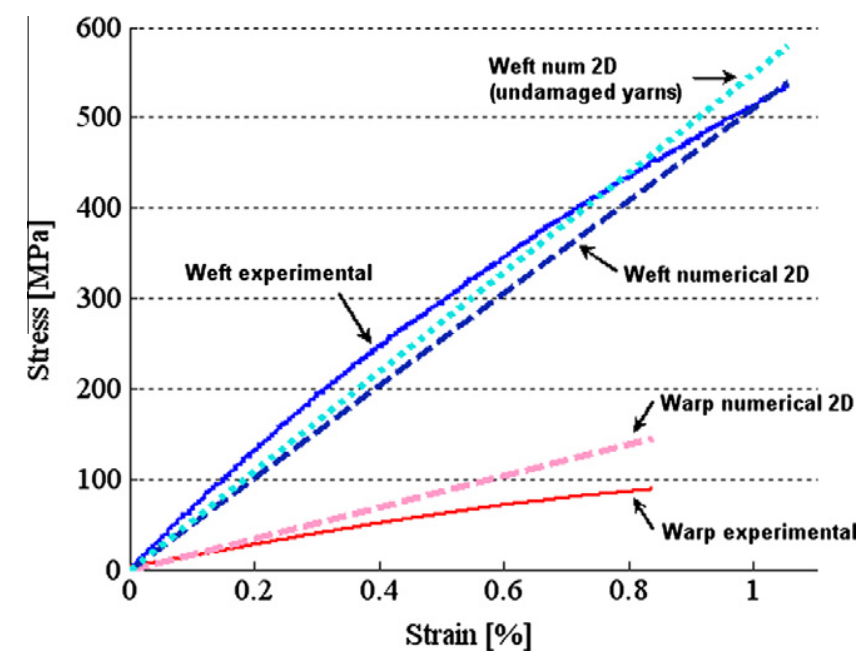

Fig. 23. Comparison of experimental and $2 \mathrm{D}$ equivalent curves under tensile load in the warp and weft directions.

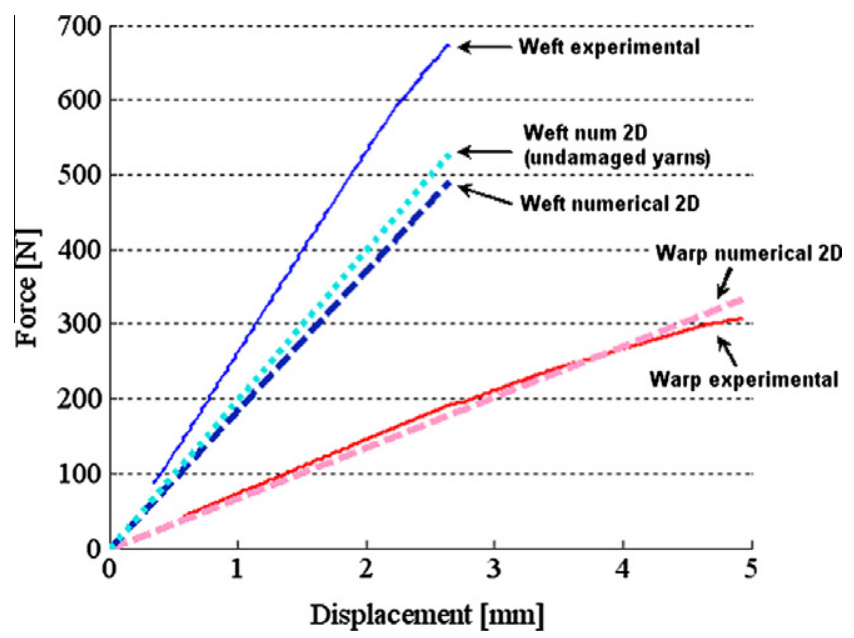

Fig. 24. Comparison of experimental and 2D equivalent curves under flexural load in the warp and weft directions.
Table 9

Tensile and bending experimental and equivalent moduli.

\begin{tabular}{llllll}
\hline & Tension & & & Bending \\
\cline { 2 - 3 } \cline { 5 - 6 } \cline { 5 - 6 } & Experimental & Numerical & & Experimental & Numerical \\
\hline Warp & $12.3 \mathrm{GPa}$ & $17.3 \mathrm{GPa}(+30 \%)$ & & $17.3 \mathrm{GPa}$ & $16.3 \mathrm{GPa}(-8 \%)$ \\
Weft & $64.5 \mathrm{GPa}$ & $51 \mathrm{GPa}(-20 \%)$ & $58 \mathrm{GPa}$ & $39.8 \mathrm{GPa}(-32 \%)$ \\
\hline
\end{tabular}

the architecture of a unit cell. The results are the effective elastic in-plane constants predicted from a periodic homogenization method. There is a good agreement of the in-plane behaviors between 3D and 2D equivalent models, except for the simple bending behavior where the transverse loadings were not included. The interlaminar shear behavior requires more advanced developments in the homogenization method, combined with short-beam experimental tests. The determination of the through-the-thickness properties is an important point for impact resistance. These elastic constants can be then used in shell-type simulations. The effective moduli are also compared to experimental results and show good agreement for the warp flexural modulus but could be improved in tension mode thanks to a better geometry representation. For the weft tensile and flexural moduli, the nesting will be one of the parameters which will increase the fiber fraction and improve the experimental comparison. Hypotheses have been introduced to ease the numerical implementation at this first stage of a more general development.

\section{Acknowledgement}

The authors thank the Nord-Pas-de-Calais Council for financial support of this work.

\section{References}

[1] Zhang C. Characterization and modeling of 3D woven composites. $\mathrm{PhD}$ thesis. USA: Graduate Faculty of North Carolina State University; 2003.

[2] Sherburn M. Geometric and mechanical modelling of textiles. PhD thesis. UK School of Mechanical, Materials and Manufacturing Engineering, University of Manchester; 2007.

[3] Cox BN, Dadkhah MS. The macroscopic elasticity of 3D woven composites. J Compos Mater 1995;29:785-819.

[4] Kuo WS, Fang J, Lin HW. Failure behavior of 3D woven composites under transverse shear. Composites: Part A 2003;34:561-75.

[5] Lee L, Rudov-Clark S, Mouritz AP, Bannister MK, Herszberg I. Effect of weaving damage on the tensile properties of three-dimensional woven composites. Compos Struct 2002;57:405-13.

[6] Callus PJ, Mouritz AP, Bannister MK, Leong KH. Tensile properties and failure mechanisms of 3D woven GRP composites. Composites: Part A 1999;30: 1277-87.

[7] Yi HL, Ding X. Conventional approach on manufacturing 3D woven preforms used for composites. J Ind Textiles 2004;34:39-50.

[8] Cox BN, Dadkhah MS, Morris WL. On the tensile failure of 3D woven composites. Composites: Part A 1996;27A:447-58.

[9] Mahadik Y, Robson Brown KA, Hallett SR. Characterisation of 3D woven composite internal architecture and effect of compaction. Composites: Part A 2010;41:872-80.

[10] Potluri P, Sagar TV. Compaction modelling of textile preforms for composite structures. Compos Struct 2008;86:177-85.

[11] Lee B, Leong KH, Herszberg I. The effect of weaving on the tensile properties of carbon fibre tows and woven composites. J Reinf Plast Compos 2001;20:652-70.

[12] Archer E, Buchanan S, Mcllhagger AT, Quinn JP, Morgan M. An investigation on the effect of 3D weaving on carbon fiber tows, fabrics and composites. In: Advani, Gillepsie, editor. TexComp 9 - Recent advances in textile composites; 2008, p. 371-9.

[13] Tan P, Tong L, Steven GP. Micromechanics models for mechanical and thermomechanical properties of 3D through-the-thickness angle interlock woven composites. Composites: Part A 1999;30:637-48.

[14] Cox BN, Carter WC, Fleck NA. A binary model of textile composites - I Formulation. Acta Metall Mater 1994;42:3463-79.

[15] Verpoest I, Lomov SV. Virtual textile composites software WiseTex: integration with micro-mechanical, permeability and structural analysis. Compos Sci Technol 2005;65:2563-74.

[16] Bogdanovich AE, Mohamed MH. Three-dimensional reinforcements for composites. SAMPE J 2009;6(45):8-28. 
[17] Boussu F, Legrand X, Serret A. General clustering of warp interlock structures. In: 1st International conference of intelligent textiles and mass customization, Casablanca, Morocco, November 15-17, 2007, p. 83-90.

[18] Rudov-Clark S, Mouritz AP, Lee L, Bannister MK. Fibre damage in the manufacture of advanced three-dimensional woven composites. Composites: Part A 2003;34:963-70.
[19] Tsai $\mathrm{KH}$, Chiu $\mathrm{CH}$, Wu TH. Fatigue behavior of 3D multi-layer angle interlock woven composite plates. Compos Sci Technol 2000;60: 241-8.

[20] Buannic N, Cartraud P, Quesnel T. Homogenization of corrugated core sandwich panels. Compos Struct 2003;59:299-312. 